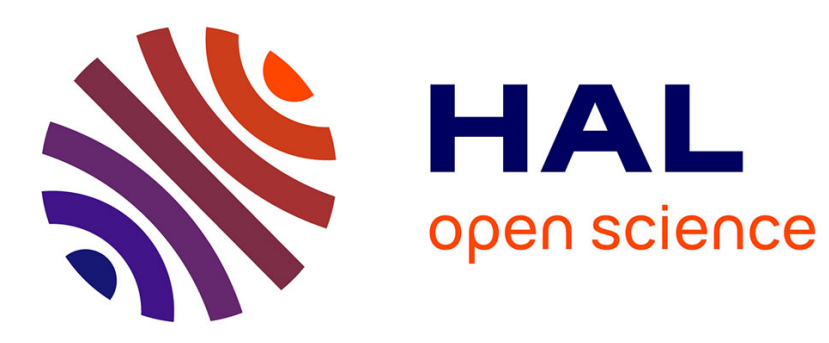

\title{
Shape of diffraction orders of centered and decentered pixelated lenses
}

Marius Peloux, Pierre Chavel, François Goudail, Jean Taboury

\section{To cite this version:}

Marius Peloux, Pierre Chavel, François Goudail, Jean Taboury. Shape of diffraction orders of centered and decentered pixelated lenses. Applied optics, 2010, 49 (6), pp.1054-1064. hal-00700311

\section{HAL Id: hal-00700311 \\ https://hal.science/hal-00700311}

Submitted on 25 May 2012

HAL is a multi-disciplinary open access archive for the deposit and dissemination of scientific research documents, whether they are published or not. The documents may come from teaching and research institutions in France or abroad, or from public or private research centers.
L'archive ouverte pluridisciplinaire HAL, est destinée au dépôt et à la diffusion de documents scientifiques de niveau recherche, publiés ou non, émanant des établissements d'enseignement et de recherche français ou étrangers, des laboratoires publics ou privés. 


\title{
Shape of diffraction orders of centered and decentered pixelated lenses
}

\author{
Marius Peloux, ${ }^{*}$ Pierre Chavel, François Goudail, \\ and Jean Taboury \\ Laboratoire Charles Fabry de I'Institut d'Optique, CNRS, Université Paris-Sud, \\ Campus Polytechnique, RD 128, 91127 Palaiseau, France \\ *Corresponding author: marius.peloux@institutoptique.fr \\ Received 22 October 2009; revised 13 January 2010; accepted 19 January 2010; \\ posted 20 January 2010 (Doc. ID 118937); published 19 February 2010
}

\begin{abstract}
We consider diffraction by pixelated lenses when the lens size is significantly smaller than the diffraction pattern of single pixels. In that case, the diffraction orders show shapes that have not been identified in earlier studies and that are quite sensitive to the pixel filling ratio as well as to decentering. (C) 2010 Optical Society of America

OCIS codes: $\quad 050.1970,070.6120,110.0110,230.6120$.
\end{abstract}

\section{Introduction}

This work originates in an unexpected observation in the diffraction pattern of a pixelated lens. Using electric circuit terminology, a pixelated lens consists in a boxcar approximation of a continuous lens shape: first, the continuous phase function is sampled over a regular grid, and then the sampled phase is assigned to the full area of the pixel. In the limit of very small pixels, the diffraction pattern of the pixelated lens would be identical to that of the initial continuous lens. However, the grating associated with the periodic arrangement of the pixels is responsible for the apparition of diffraction orders.

Using a pixelated lens that had been fabricated by photolithography, we observed that while in some cases the diffraction orders observed in the focal plane were, as we had intuitively expected, fairly similar to the Fraunhofer diffraction pattern by the pupil, in some other situations a notable change occurred and a central minimum appeared in the diffraction pattern, which would be more similar to the derivative of the initial pupil diffraction pattern that to that pattern itself. This happened in particular in

0003-6935/10/061054-11\$15.00/0

(C) 2010 Optical Society of America the case of major practical interest of a well-centered pixelated lens.

Earlier publications introduced pixelated lenses as diffractive elements that can be displayed on a spatial light modulator [1], leading to moderate-resolution pixelated lenses. The shape and intensity of the diffraction orders by those components, responsible for a multiple imaging effect, were already analyzed in several publications [2-4] , and a precise description of the central diffraction order was made [5], revealing a peculiar self-apodization effect. In our case, the photolithographic equipment used allowed us to reach a fairly high quality, and the effect observed turns out to be different.

It is appropriate here to stress that the component of interest to us, while affected by diffraction indeed, is a pixelated lens but not a diffractive lens (see Fig. 1). By "diffractive," we mean that the design of the element resorts to phase wrapping, introducing zones and phase jumps, as is often necessary when displaying phase functions on a spatial light modulator [Fig. 1(b)]. The phase jumps are typically an integer number of $2 \pi$ at some appropriate design wavelength. The spatial light modulator pixels in addition define regions of constant phase, so that those lenses are both diffractive and pixelated [Fig. 1(d)]. In our case, no phase wrapping was needed. While the lens does consist in a set of closely packed pixels 
[Fig. 1(c)], no diffractive zones exist. Instead, the height of each pixel is identical to that of the initial continuous lens at the sampling point in the center of the pixel. We study only the influence of the spatial discretization of the phase distribution of the lens and not the quantization and discontinuities of the phase value $[6,7]$.

While the analytical approach of [5] is perfectly applicable to our situation, it turns out that our observation of a derivation effect in all orders, save for the central order, of a well-centered pixelated lens is more appropriately described and physically understood by a different analytical approach. That is the purpose of the present paper.

We consider applications where the multiple imaging effect is not desirable; then for a given pixel size and pupil size we choose a larger focal length to reduce this effect. However, some diffraction orders remain and can introduce perturbations in images formed by such lenses. It is thus useful to evaluate their shape and their efficacy. We perform this analysis in two cases: when the phase function of the lens is centered on the pupil and when it is decentered with respect to one of the two main diffraction directions determined by the symmetries in the pixel arrangement.

The paper is organized as follows: in Section 2, we present a mathematical approach describing the diffraction of light by a pixelated lens, based on Fourier optics. In Section 3 , we analyze the shape and efficiency of the diffraction orders when the phase distribution of the lens is centered with respect to its pupil and illustrate the results on an image obtained with a pixelated lens fabricated by photolithography. Finally, in Section $\underline{4}$ we analyze the effect of pupil decentering.

\section{Basic Mathematical Formulation}

\section{A. Notation}

We consider a thin pixelated lens, whose pixel pitch $p$, and whose pixels are $a \times a$ squares, with obviously

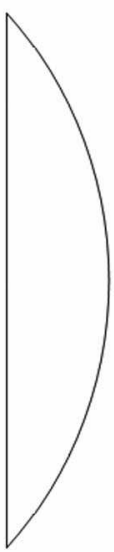

(a)

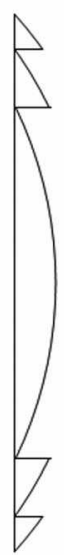

(b)

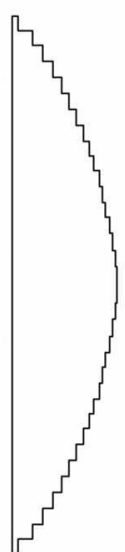

(c)

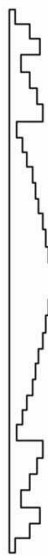

(d)
Fig. 1. (a) Continuous lens. (b) Diffractive lens. (c) Pixelated lens. (d) Diffractive pixelated lens.
$0 \leq a \leq p$. The repartition of the centers of the pixels is periodic along the $x$ and $y$ axes, which are the two main diffraction directions. The pixel profile is denoted $\Pi_{2, a}(\vec{r})=\Pi_{2}(\vec{r} / a)$, where $\Pi_{2}$ is the $2 \mathrm{D}$ rectangular function, and $\vec{r}=(x, y)$ is the position vector. The transmittance of the pixelated lens is obtained by sampling the transmittance $t_{c}(\vec{r})$ of a thin refractive lens in the plane $z=0$, denoted $\Sigma_{1}$ :

$$
t_{c}(\vec{r})=P(\vec{r}) \exp \left(-i \frac{\pi r^{2}}{\lambda f}\right)
$$

The index $c$ stands for continuous. The parameter $f$ is the focal length, and $\lambda$ the design wavelength. In this paper, we shall consider only monochromatic illumination of wavelength $\lambda$. The lens whose transmittance is $t_{c}(\vec{r})$ will be called the equivalent refractive lens (ERL). $P$ is the pupil function of the lens. In the case where the pupil is square and centered, $P(\vec{r})=\Pi_{2}(\vec{r} / N p)$, where $N$ is the number of pixels along $x$ or $y$.

We suppose that the pixelated lens is illuminated by a plane wave. We compute the amplitude of the diffracted wave in plane $z=f$, denoted $\Sigma_{2}$, where we expect to see the focus. The observed diffraction pattern is a coherent sum of the diffraction patterns of all pixels. We let $\vec{\rho}=(\xi, \eta)$ denote the $2 \mathrm{D}$ coordinates in plane $\Sigma_{2}$. Pixels are indexed by the $2 \mathrm{D}$ vector $\vec{j}=\left(j_{x}, j_{y}\right)$, whose coordinates are integers [8]. The coordinates of the center of pixel $\vec{j}$ are $\left(j_{x} p, j_{y} p\right)$. Figure 2 illustrates the notation.

We consider the sampled transmittance in plane $(\vec{r}, z=0)$, starting from samples in the mathematical sense, i.e. Dirac distributions $\delta$ :

$$
t_{\Delta}(\vec{r})=t_{c}(\vec{r}) \amalg_{p, p}(\vec{r}),
$$

where $\amalg_{p, p}$ is a $2 \mathrm{D}$ Dirac comb:

$$
\begin{aligned}
\forall(x, y) & \in \mathbb{R}^{2}, \amalg_{p, p}(x, y) \\
& =\sum_{n_{x}=-\infty}^{+\infty} \delta\left(x-n_{x} p\right) \times \sum_{n_{y}=-\infty}^{+\infty} \delta\left(y-n_{y} p\right) .
\end{aligned}
$$

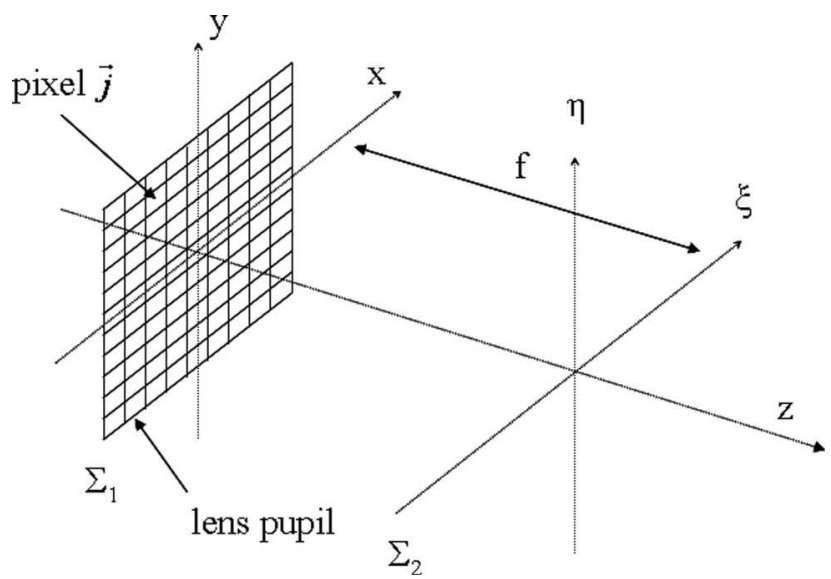

Fig. 2. Scheme illustrating the notation used in the basic mathematical formulation. 
In a pixelated lens, each sample of $t_{c}$ is in fact the center of a uniform pixel whose transmittance is equal to the sampled value. If $U_{0}$ is the amplitude of the incident plane wave, the complex amplitude in the plane $\Sigma_{1}$ of the pixelated lens is $U_{0} t(\vec{r})$ with

$$
t(\vec{r})=t_{\Delta}(\vec{r}) * \Pi_{2, a}(\vec{r}) .
$$

\section{B. Assumptions about Fresnel Numbers}

Here we introduce the Fresnel generalized number [9] $(\mathrm{FGN})$ :

$$
\mathrm{FGN}=\frac{r_{0}^{2}}{\lambda f},
$$

where $r_{0}$ is the radius of the smallest circular pupil that contains the pupil of the pixel. As the pixel pupil is an $a \times a$ square, $r_{0}=a \sqrt{2} / 2$. We assume that the FGN for one pixel is much smaller than typically 1 , which is a common criterion for the validity of the Fraunhofer approximation. This hypothesis will be called $\left(H_{1}\right)$ and will be written as follows:

$$
\text { Hypothesis }\left(H_{1}\right): \text { FGN }=\frac{a^{2}}{2 \lambda f} \ll 1 .
$$

In this study, our concern is about pixels whose size $a \leq p \leq 100 \mu \mathrm{m}$ and focal lengths $f \geq 10 \mathrm{~cm}$; then in visible light $\left(H_{1}\right)$ is easily verified, since $a^{2} / 2 \lambda f$ cannot exceed 0.1 for $\lambda=0.5 \mu \mathrm{m}$. We will thus assume in the rest of the paper that we are in the Fraunhofer diffraction regime for pixel diffraction and that we can write the Fresnel integral using the Fraunhofer approximation for the central pixel diffraction at the distance $f$ :

$$
u_{\overrightarrow{0}}(\vec{\rho})=-i \frac{U_{0}}{\lambda f} t_{c}(\overrightarrow{0}) \exp \left(2 \pi i \frac{f}{\lambda}+i \pi \frac{\|\vec{\rho}\|^{2}}{\lambda f}\right) \tilde{\Pi}_{2, a}\left(\frac{\vec{\rho}}{\lambda f}\right) .
$$

$\tilde{\Pi}_{2, a}$ is the Fourier transform of $\Pi_{2, a}: \tilde{\Pi}_{2, a}(\vec{\rho} / \lambda f)=$ $a^{2} \operatorname{sinc}(a \xi / \lambda f) \operatorname{sinc}(a \eta / \lambda f)$. The diffracted field associated with the pixel $\vec{j}$ is obtained by translation:

$$
\begin{aligned}
u_{\vec{j}}(\vec{\rho})= & -i \frac{U_{0}}{\lambda f} t_{c}(\vec{j} p) \exp \left(2 \pi i \frac{f}{\lambda}+i \pi \frac{\|\vec{\rho}-\vec{j} p\|^{2}}{\lambda f}\right) \\
& \times \tilde{\Pi}_{2, a}\left(\frac{\vec{\rho}-\vec{j} p}{\lambda f}\right) .
\end{aligned}
$$

We introduce the following auxiliary function:

$$
f_{\vec{j}}(\vec{\rho}) \triangleq P(\vec{j} p) \exp \left(-2 i \pi \frac{\vec{j} p \cdot \vec{\rho}}{\lambda f}\right) \tilde{\Pi}_{2, a}\left(\frac{\vec{\rho}-\vec{j} p}{\lambda f}\right)
$$

Then, from Eq. (1),

$$
u_{\vec{j}}(\vec{\rho})=-i \frac{U_{0}}{\lambda f} \exp \left[\frac{2 \pi i}{\lambda}\left(f+\frac{\|\vec{\rho}\|^{2}}{2 f}\right)\right] f_{\vec{j}}(\vec{\rho})
$$

The complex amplitude in the plane $\Sigma_{2}$ results from the interferences between the diffraction patterns of all pixels and is thus $\sum_{\vec{j} \in(\mathbb{Z} \mathbb{Z})} u_{\vec{j}}(\vec{\rho})$. Since the term preceding $f_{\vec{j}}(\vec{\rho})$ in Eq. (8) is not pixel dependant, we focus on $\sum_{\vec{j} \in(\mathbb{Z}, \mathbb{Z})} f_{\vec{j}}(\vec{\rho})$. We have

$$
\begin{aligned}
S(\vec{\rho})= & \sum_{\vec{j} \in \mathbb{Z}^{2}} f_{\vec{j}}(\vec{\rho})=\sum_{\vec{j} \in \mathbb{Z}^{2}} P(\vec{j} p) \exp \left(-2 \pi i \frac{\vec{j} p \cdot \vec{\rho}}{\lambda f}\right) \\
& \times \tilde{\Pi}_{2, a}\left(\frac{\vec{\rho}-\vec{j} p}{\lambda f}\right) .
\end{aligned}
$$

We introduce a second assumption:

$$
\text { Hypothesis }\left(H_{2}\right): \frac{A p}{\lambda f} \ll 1 \text {, }
$$

where $A=N p$ is the lens pupil width along the $x$ or $y$ axis. We assume that the lens pupil is contained in an $A \times A$ square. The ERL point spread function (PSF) width is then of the order of $\lambda f / A$. Then $\left(H_{2}\right)$ means physically that the pixel pitch $p$ is much smaller than the width of the PSF associated with the ERL. We note that if $\left(H_{2}\right)$ is verified, then $\left(H_{1}\right)$ is also verified, since obviously $N p^{2} \geq a^{2} / 2$. Moreover $\left(H_{2}\right)$ implies, for any pixel $j$ inside the pupil, $\|\vec{j}\| p / \lambda f \leq A / \lambda f \ll$ $1 / p . \Pi_{2 . a}$ is contained in a $p \times p$ square. Its Fourier transform $\tilde{\Pi}_{2 . a}$ is therefore wider than $1 / p$. In the case where the pixels are $a \times a$ squares with $p=a$, $1 / p$ is exactly the central lobe half-width of $\Pi_{2, a}$.

One may comment that the FGN for the whole lens pupil $A^{2} / 2 \lambda f$, as opposed to that of a single pixel, is not smaller than unity. For a lens to show a substantial focusing power, it should on the contrary be larger than unity. Hypothesis $\left(H_{2}\right)$ assumes that the geometrical average between the pixel FGN and the lens FGN is significantly smaller than unity. In other words, whereas $\left(H_{1}\right)$ amounts to assuming the pixel size to be much smaller than its own diffraction pattern in the focal plane, $\left(H_{2}\right)$ relates to the case where the whole lens size is much smaller than the pixel diffraction pattern. This assumption will now be used to derive a physical interpretation of the diffraction order built up by the interference between pixel diffraction patterns of Eq. ( $\underline{9})$.

\section{Expanding the Diffraction Pattern of Individual Pixels}

For simplicity's sake, we restrict the following calculation in this section to one dimension, but it can be easily extended to two (see Appendix A). The 1D rectangular function of width $a$ is denoted $\Pi_{1, a}$, and its Fourier transform is denoted $\tilde{\Pi}_{1, a} .\left(H_{2}\right)$ implies that the variations of $\tilde{\Pi}_{1, a}$ between $\xi / \lambda f$ and $(\xi-j p) / \lambda f$ are small enough to assume that $\Pi_{1, a}[(\xi-j p) / \lambda f]$ can be approximated by its first-order Taylor expansion around $\xi / \lambda f$ :

$$
\tilde{\Pi}_{1, a}\left(\frac{\xi-j p}{\lambda f}\right) \simeq \tilde{\Pi}_{1, a}\left(\frac{\xi}{\lambda f}\right)-\frac{j p}{\lambda f} \Pi_{1, a}^{\prime}\left(\frac{\xi}{\lambda f}\right)
$$


In other words, the diffraction pattern by one pixel in plane $\Sigma_{2}$ can be expanded linearly over a distance of the order of the pixelated lens pupil. Then, if $\amalg_{p}$ is a 1D Dirac comb,

$$
\forall x \in \mathbb{R}, \amalg_{p}(x)=\sum_{n_{x}=-\infty}^{+\infty} \delta\left(x-n_{x} p\right) .
$$

Equation (9) becomes, after straightforward algebra,

$$
\begin{aligned}
S(\xi)= & \mathrm{FT}\left[P(x) \amalg_{p}(x)\right]_{\xi / \lambda f} \tilde{\Pi}_{1, a}\left(\frac{\xi}{\lambda f}\right) \\
& -\frac{1}{\lambda f} \mathrm{FT}\left[x P(x) \amalg_{p}(x)\right]_{\xi / \lambda f} \tilde{\Pi}_{1, a}^{\prime}\left(\frac{\xi}{\lambda f}\right),
\end{aligned}
$$

where FT stands for Fourier transform. Another way to write Eq. (12) is

$$
\begin{aligned}
S(\xi)= & \left(\tilde{P} * \amalg_{1 / p}\right)_{\xi / \lambda f} \tilde{\Pi}_{1, a}\left(\frac{\xi}{\lambda f}\right) \\
& +\frac{1}{2 \pi i \lambda f}\left(\tilde{P}^{\prime} * \amalg_{1 / p}\right)_{\xi / \lambda f} \tilde{\Pi}_{1, a}^{\prime}\left(\frac{\xi}{\lambda f}\right) .
\end{aligned}
$$

Equation (13) can also be written as

$$
S(\xi)=S_{A}(\xi)+S_{B}(\xi)
$$

with

$S_{A}(\xi)=\sum_{n \in \mathbb{Z}} S_{A, n}\left(\frac{\xi}{\lambda f}\right)=\tilde{\Pi}_{1, a}\left(\frac{\xi}{\lambda f}\right) \sum_{n \in \mathbb{Z}} \tilde{P}\left(\frac{\xi}{\lambda f}-\frac{n}{p}\right)$,

$S_{B}(\xi)=\sum_{n \in \mathbb{Z}} S_{B, n}\left(\frac{\xi}{\lambda f}\right)=\frac{\tilde{\Pi}_{1, a}^{\prime}\left(\frac{\xi}{\lambda f}\right)}{2 \pi i \lambda f} \sum_{n \in \mathbb{Z}} \tilde{P}^{\prime}\left(\frac{\xi}{\lambda f}-\frac{n}{p}\right)$.

$S_{A}(\xi)$ is a sum of the lens pupil diffraction patterns located at each diffraction order $n$ of the pixel grating, modulated by the pixel diffraction pattern. $S_{B}(\xi)$ is a sum of the derivatives of the lens pupil diffraction patterns located at each diffraction order $n$ of the pixel grating, modulated by the derivative of the pixel diffraction pattern in the $\xi$ direction.

We also use the following notation:

$$
S(\xi)=\sum_{n \in \mathbb{Z}} S_{n}\left(\frac{\xi}{\lambda f}\right)
$$

with $\forall n \in \mathbb{Z}, S_{n}\left(\frac{\xi}{\lambda f}\right)=S_{A, n}\left(\frac{\xi}{\lambda f}\right)+S_{B, n}\left(\frac{\xi}{\lambda f}\right)$.

To summarize, in this section, we have written $S(\xi)$ as a sum of diffraction patterns associated with diffraction orders, after having first expanded $\tilde{\Pi}_{1 . a}[(\xi-$ $j p) / \lambda f]$ to first order around $\xi / \lambda f$ and written Eq. (9) in terms of Fourier transforms; this led us to an expression including convolution by a Dirac comb. Each Dirac function of the comb stands for a diffraction order, whose shape will be analyzed in the following sections. In Section 3 we study the case where the pixelated lens is centered in the pupil, and in Section 4 we study the case where this lens is decentere $\bar{d}$ in the pupil.

\section{Shape of Diffraction Orders for a Pixelated Lens Centered in the Pupil}

\section{A. Analysis}

Still considering the 1D case, the expression of the pupil is $P(x)=\Pi_{1, A}(x)$. We note that $\operatorname{sinc}(x)=$ $\sin (\pi x) / \pi x$. Then

$$
\begin{aligned}
& S_{A}(\xi)=a \operatorname{sinc}\left(\frac{\xi}{\lambda f} a\right) \sum_{n \in \mathbb{Z}} A \operatorname{sinc}\left[\left(\frac{\xi}{\lambda f}-\frac{n}{p}\right) A\right], \\
& S_{B}(\xi)=\frac{a^{2}}{2 \pi i \lambda f} \operatorname{sinc}^{\prime}\left(\frac{\xi}{\lambda f} a\right) \sum_{n \in \mathbb{Z}} A^{2} \operatorname{sinc}^{\prime}\left[\left(\frac{\xi}{\lambda f}-\frac{n}{p}\right) A\right] .
\end{aligned}
$$

In Figure 3, we show the shape of the sinc function and the shape of its derivative.

We notice in Eqs. (15) that the $S_{B}$ to $S_{A}$ weighting factor ratio is $a A / 2 \overline{\pi \lambda f}$, which is low according to $\left(H_{2}\right)$. We will analyze the shape and efficiency of the diffraction orders, considering two cases: the case where $a=p$ and the case where $a \neq p$.
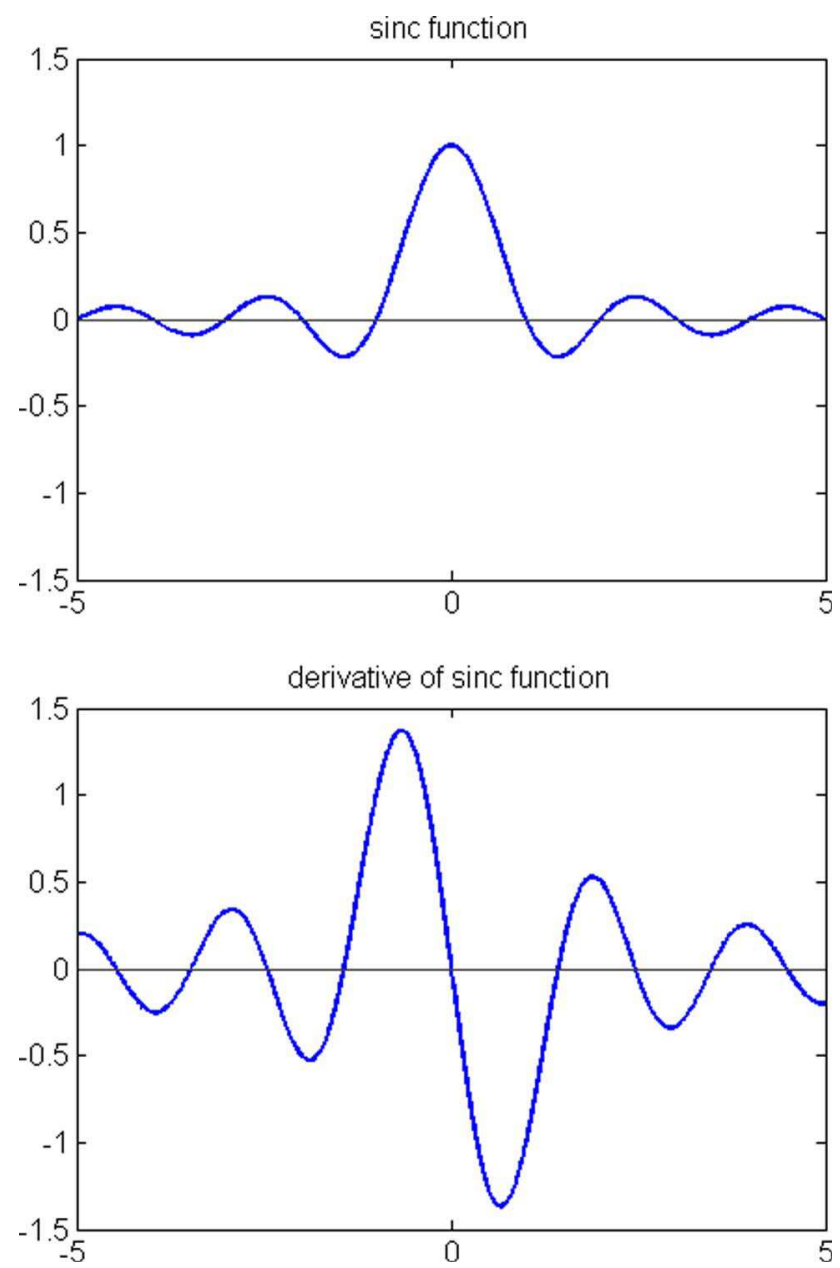

Fig. 3. (Color online) Shape of the sinc function and shape of the derivative of the sinc function. 


\section{Case $\mathrm{a}=\mathrm{p}$}

Let us first consider $a=p$. In this case,

$$
\begin{aligned}
& S_{A}(\xi)=p \operatorname{sinc}\left(\frac{\xi}{\lambda f} p\right) \sum_{n \in \mathbb{Z}} A \operatorname{sinc}\left[\left(\frac{\xi}{\lambda f}-\frac{n}{p}\right) A\right] \\
& S_{B}(\xi)=\frac{p^{2}}{2 \pi i \lambda f} \operatorname{sinc}^{\prime}\left(\frac{\xi}{\lambda f} p\right) \sum_{n \in \mathbb{Z}} A^{2} \operatorname{sinc}^{\prime}\left[\left(\frac{\xi}{\lambda f}-\frac{n}{p}\right) A\right]
\end{aligned}
$$

For $n \neq 0$, and if $\xi / \lambda f$ is close to $n / p, \operatorname{sinc}(p \xi / \lambda f) \simeq 0$. where $t(x)=t_{\Delta}(x) * \Pi_{1, a}(x)$ is the transmittance of the lens and $u(\xi, f)$ is the repartition of the complex amplitude in the plane $\Sigma_{2}$.

Then we compare the results obtained with the analytical expressions describing the shape of the diffraction orders determined in Subsection 3.A. We show intensity patterns, which means that we consider the square modulus of the complex amplitude.

We will consider a pixelated lens whose characteristics are $A=4 \mathrm{~mm}, p=10 \mu \mathrm{m}, \lambda=500 \mathrm{~nm}, f=1 \mathrm{~m}$. $\left(H_{2}\right)$ is clearly verified $(A p / \lambda f=0.08)$. The

So

$$
\begin{gathered}
S_{0}(\xi)=S_{A, 0}(\xi)+S_{B, 0}(\xi) \simeq S_{A, 0}(\xi)=p A \operatorname{sinc}(p \xi / \lambda f) \operatorname{sinc}(A \xi / \lambda f) \\
\forall n \neq 0, S_{n}(\xi) \simeq S_{B, n}(\xi)=\left(p^{2} \operatorname{sinc}^{\prime}(p \xi / \lambda f) / 2 \pi i \lambda f\right) A^{2} \operatorname{sinc}^{\prime}[(\xi / \lambda f-n / p) A]
\end{gathered}
$$

As $A \gg p$, the modulating factors $\operatorname{sinc}(p \xi / \lambda f)$ and $\operatorname{sinc}^{\prime}(p \xi / \lambda f)$ have slow variations in comparison with $\operatorname{sinc}(A \xi / \lambda f)$ and $\operatorname{sinc}^{\prime}(A \xi / \lambda f)$. This means that the diffraction pattern associated with the central order $n=0$ is a sinc function of width $\lambda f / A$ and that the diffraction patterns associated with other orders have the shape of the derivative of a sinc function.

\section{Case $\mathrm{a} \neq \mathrm{p}$}

Let us now consider that $a \neq p$, which means in fact $a<p$. For every $n$, and if $\xi / \lambda f$ is close to $n / p$, $\operatorname{sinc} a \xi / \lambda f \neq 0$. Moreover, as $\left(H_{2}\right)$ implies $a A / 2 \pi \lambda f$ $\ll 1$, then $S \simeq S_{A}$, and the diffraction orders are sinc functions:

$$
\begin{aligned}
\forall n & \in \mathbb{Z}, S_{n}(\xi) \simeq S_{A, n}(\xi) \\
& =a \operatorname{sinc}\left(\frac{\xi}{\lambda f} a\right) A \operatorname{sinc}\left[\left(\frac{\xi}{\lambda f}-\frac{n}{p}\right) A\right] .
\end{aligned}
$$

The efficiency of each diffraction order normalized to the central order is approximately $\operatorname{sinc}(n a / p)$, whereas in the case $a=p$ it is $|A p \operatorname{sinc}(n) / 2 \pi \lambda f|=$ $A p / 2 n \pi \lambda f$ for the order $n, n \neq 0$. This means that if $a \neq p$, the nonzero orders are more significant in relation to the zero order than if $a=p$.

\section{B. Examples}

We want to illustrate that the expressions describing the shapes and efficiencies of the diffraction orders determined via our approximations are valid. Therefore, we simulate the diffraction patterns of some pixelated lenses by direct calculation via Fresnel approximation:

$u(\xi, f)=-i \frac{U_{0}}{\lambda f} \exp \left(2 \pi i \frac{f}{\lambda}\right) \int_{\mathbb{R}} t(x) \exp \left(+i \pi \frac{(x-\xi)^{2}}{\lambda f}\right) \mathrm{d} x$, diffraction order $n$ is located around $\xi=n \lambda f / p=n \times$ $5 \times 10^{-2} \mathrm{~m}$.

In the case where $a=p$, we represent in Table 1 the normalized intensity of the diffraction pattern in the focal plane around the orders 0,1 , and 2 . Except for order 0, we give three values. The central value is the normalized intensity at the center of order $n$. The other two are the local maxima around that center.

The efficiency of orders 1 and 2 are very low in comparison with the efficiency of order 0 , as already stated for the case where $a=p$. We represent in Fig. 4(a) the shape of the central order, calculated by direct calculation via Eq. (18). When we compare this curve to that obtained via Eq. (16), we see that these curves are almost perfectly superimposed at the scale shown. We note the maximal error

$$
\Delta=\max _{\xi \in D}|f(\xi)-g(\xi)|,
$$

where $f(g)$ is the function obtained by direct calculation ( via the approximations made in Subsection 3.A) and $D$ is the definition domain of $f$ and $g$. In this case $\Delta=2.75 \times 10^{-4}$, which reveals that $S_{0}(\xi) \simeq S_{A, 0}(\xi)$ is a good approximation for describing the shape of the central order.

Now we study the case where $a=p=200 \mu \mathrm{m}$; then $\left(H_{2}\right)$ is not verified $(A p / \lambda f=1.6)$. Indeed, in Fig. $4(\mathrm{~b})$ one can see that the curve obtained by direct calculation is different from the one obtained via the approximations made in Subsection 3.A, and in this case $\Delta=1.60 \times 10^{-1}$. As was already investigated in some detail in [5], we observe the apodization of the noncentral lobes of the PSF pattern of the ERL, which is here a simple sinc function.

Let us now consider order 1 . Curves normalized to the local maximum are shown in Fig. 5(a), in the case where $a=p=10 \mu \mathrm{m}$. We compare the curve obtained by direct calculation via the Fresnel approximation to the one obtained via Eq. (16). Here again 
Table 1. Normalized Intensity of Orders 0,1 , and 2

\begin{tabular}{|c|c|c|c|c|c|c|c|}
\hline \multirow[b]{2}{*}{ Quantity } & \multicolumn{7}{|c|}{ Order } \\
\hline & 0 & & 1 & & & 2 & \\
\hline$\xi(\mathrm{mm})$ & 0 & 49.92 & 50 & 50.08 & 99.92 & 100 & 100.08 \\
\hline Normalized intensity & 1 & $3.06 \times 10^{-4}$ & $2.85 \times 10^{-7}$ & $3.02 \times 10^{-4}$ & $7.62 \times 10^{-5}$ & $1.78 \times 10^{-8}$ & $7.57 \times 10^{-5}$ \\
\hline
\end{tabular}

the curves associated with $f$ and $g$ match quasi perfectly, as $\Delta=4.13 \times 10^{-3}$.

Finally, we consider the case where $a=$ $p=200 \mu \mathrm{m}$. We represent the shape of the first order, located around $\xi=2.5 \times 10^{-3} \mathrm{~m}$ in Fig. 5(b). We verify that the curves associated with $f$ and $g$ do not match in this case, where $\left(H_{2}\right)$ is not clearly verified: $\Delta=3.18 \times 10^{-1}$. Nevertheless, our approximation still provides a fair first grasp at the phenomenon.

Let us now consider the case where $a \neq p$. Choosing again $p=10 \mu \mathrm{m}$, we observe in Fig. 6 the diffraction pattern associated with order 1 obtained with $a=$ $p / 2=5 \mu \mathrm{m}$. The curves associated with $f$ and $g$ are obtained, respectively, via Eqs. (17) and (18) match: $\Delta=3.50 \times 10^{-4}$.

In this section, where the pixelated lens is centered on the pupil, we verified through different numerical examples that the approximations made in Subsection 3.A are reasonable when $\left(H_{2}\right)$ is verified,
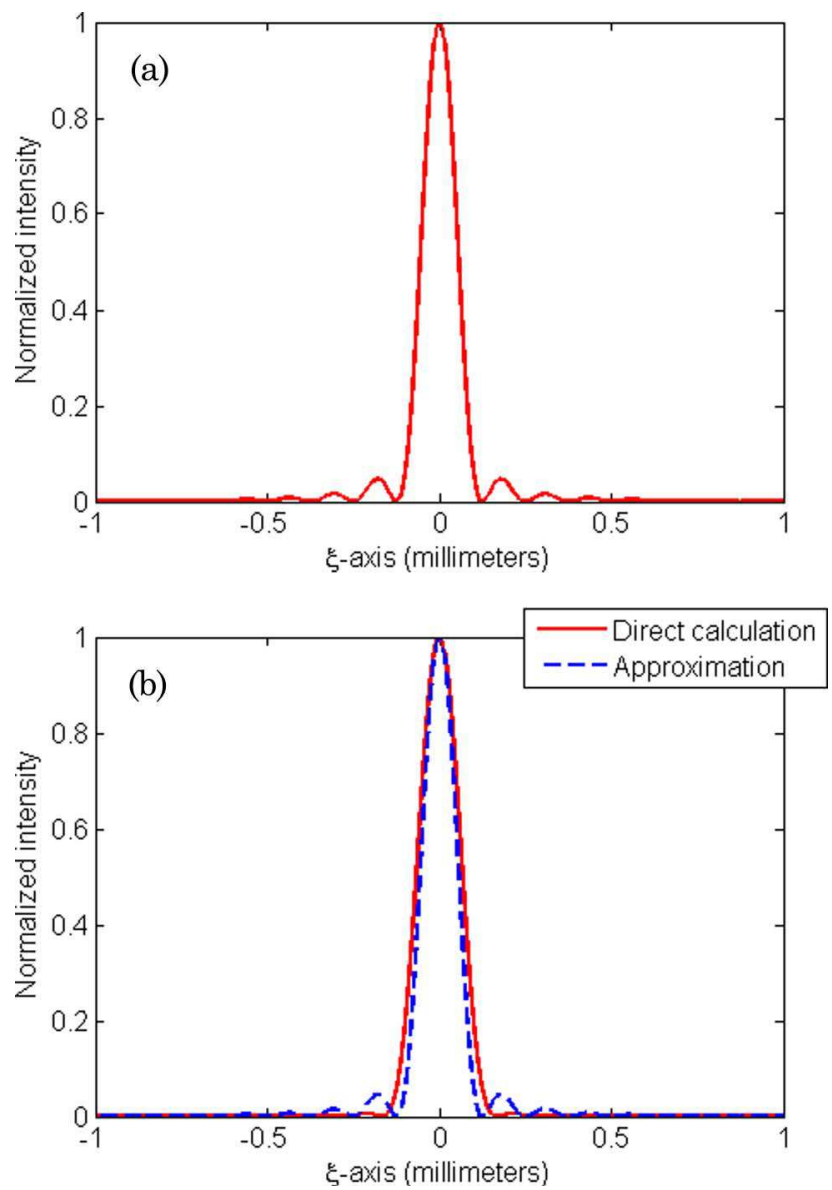

Fig. 4. (Color online) Central order of a centered pixelated lens: (a) $a=p=10 \mu \mathrm{m}$, (b) $a=p=200 \mu \mathrm{m}$. as the error function $\Delta$ is smaller than $1 \%$ in all cases studied.

\section{Experimental Results}

The experiment described here is intended to illustrate the results obtained in Subsection 3.A. A2D pixelated lens was fabricated with the characteristics $f=1 \mathrm{~m}$ and $a=p=20 \mu \mathrm{m}$, and the side length of the square pupil of this lens is $A=2 \mathrm{~mm}$. We illuminated this lens with an expanded $\mathrm{He}-\mathrm{Ne}$ laser: $\lambda \simeq 633 \mathrm{~nm} .\left(H_{2}\right)$ is verified as $(A p / \lambda f \simeq 0.06)$. Figure 7 shows the diffraction pattern, observed with a linear camera, with the central order considerably saturated in spite of the fact that the central part of the figure has been dimmed, and the first orders in the $x$ and $y$ directions. The size of Figure 7 is $70 \mathrm{~mm} \times 70 \mathrm{~mm}$. As the dynamic range of the camera used for the photograph was not large enough to clearly show the shape of the zero and nonzero orders simultaneously, we took pictures of the diffraction orders separately, with
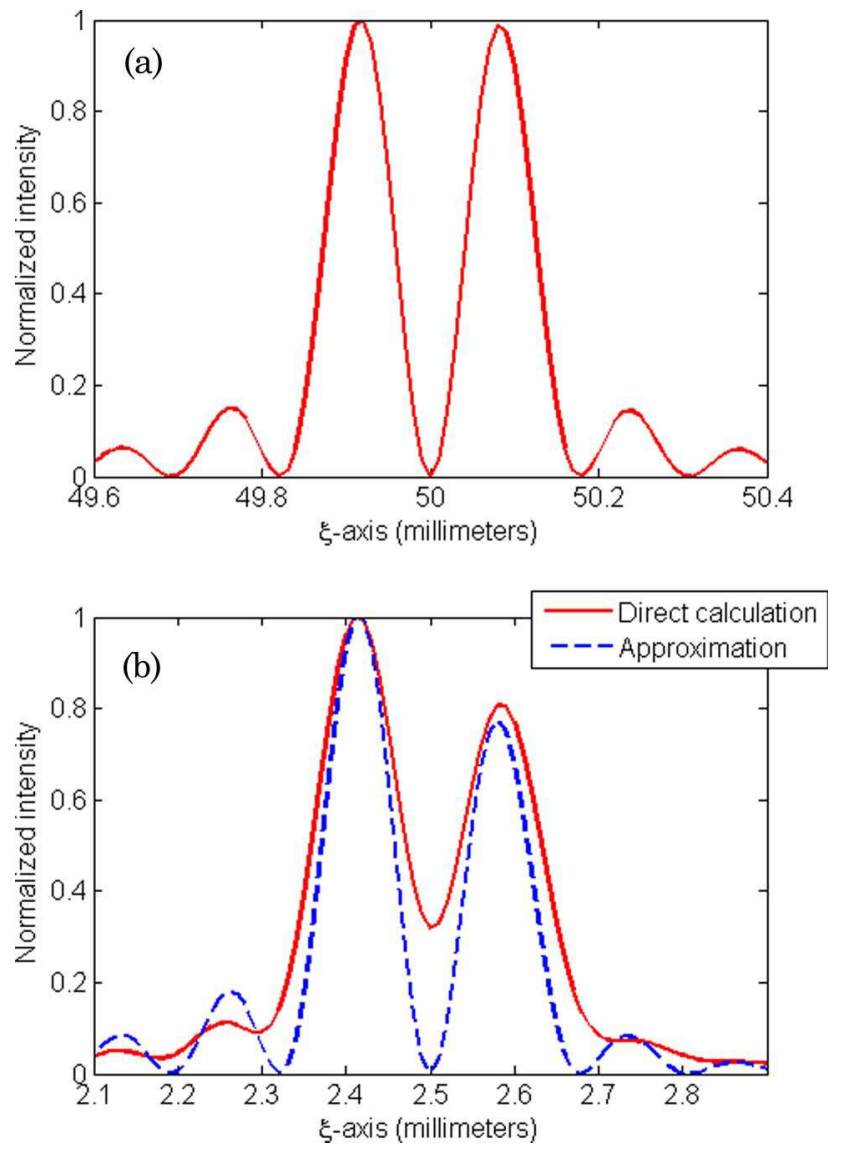

Fig. 5. (Color online) First order of a centered pixelated lens: (a) $a=p=10 \mu \mathrm{m}$, (b) $a=p=200 \mu \mathrm{m}$. 


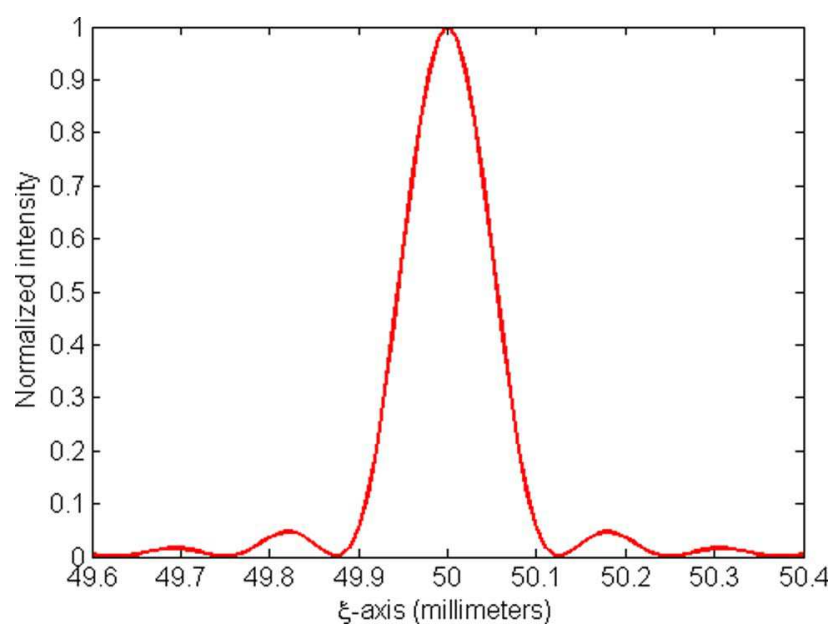

Fig. 6. (Color online) First order of a centered pixelated lens: $a=p / 2=5 \mu \mathrm{m}$.

different exposure times. Then, Figure 8 shows more precisely the shape of the central order. One easily identifies the product of two sinc functions. Figure 9 is a magnified version of the zone inside the upper solid square in Fig. 7, i.e., order $\vec{j}=(0,1)$. The size of Fig. 8 and 9 is $10 \mathrm{~mm} \times 10 \mathrm{~mm}$. We also show in Fig. 10 the vertical cross section of the diffraction order shown in Fig. 9 , and we compare it with the square modulus of the derivative of a sinc function with the appropriate parameters. The similitude between measure and approximation confirms the results obtained in Subsection 3.A.

\section{Decentered Pixelated Lens}

\section{A. Analysis}

We propose to study the effect of decentering the lens pupil along the $x$ direction inside the pupil plane, which is equivalent to translating the phase function of the lens in relation to a fixed pupil. $X$ is the translation of the center of the lens along $x$.

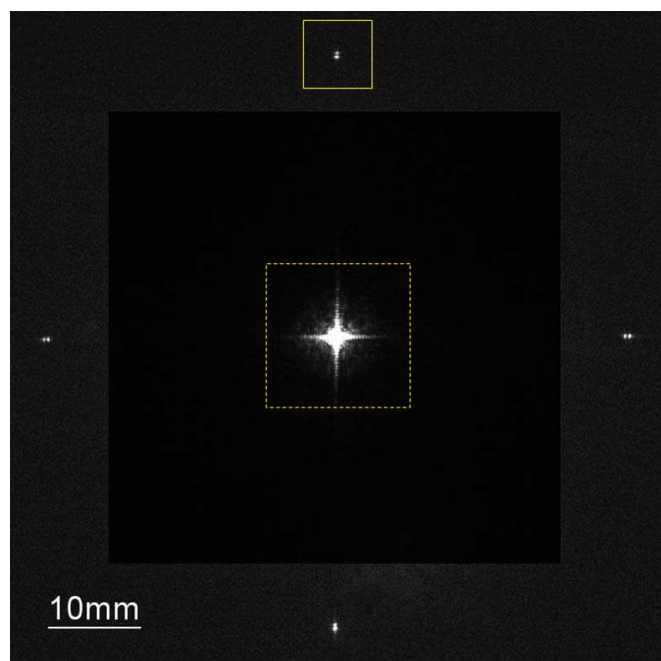

Fig. 7. (Color online) Observation of the repartition of light in the focal plane of a pixelated lens. Dashed frame, the central order; solid frame, order $(0,1)$.

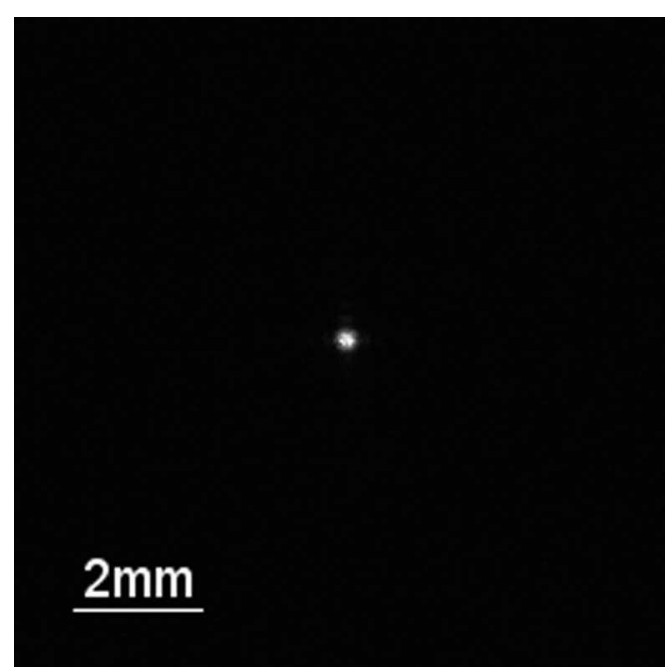

Fig. 8. Observation of the shape of the central order.

Equation (1) in one dimension then becomes $t_{c}(x)=\Pi_{1, A}(x) \exp \left(-i \pi(x-X)^{2} / \lambda f\right)$, which leads us to consider a different expression for $S$ :

$$
\begin{aligned}
& S_{A}(\xi)=a \operatorname{sinc}\left(a \frac{\xi}{\lambda f}\right) \sum_{n \in \mathbb{Z}} A \operatorname{sinc}\left[\left(\frac{\xi}{\lambda f}-\frac{X}{\lambda f}-\frac{n}{p}\right) A\right], \\
& S_{B}(\xi)=\frac{a^{2}}{2 \pi i \lambda f} \operatorname{sinc}^{\prime}\left(\frac{\xi}{\lambda f} a\right) \sum_{n \in \mathbb{Z}} A^{2} \operatorname{sinc}^{\prime}\left[\left(\frac{\xi}{\lambda f}-\frac{X}{\lambda f}-\frac{n}{p}\right) A\right] .
\end{aligned}
$$

We show how to get this result in Appendix B. The diffraction pattern associated with the order $n$ is located around $\xi=n \lambda f / p+X$.

We will consider two cases that depend on the value of $X$.

\section{Lens Decentering an Integer Multiple of the Diffracted Order Spacing}

If $\exists n_{X}, \quad-X / \lambda f=n_{X} / p$, i.e., if the lens decentering in the pupil is an integer multiple of the diffracted order

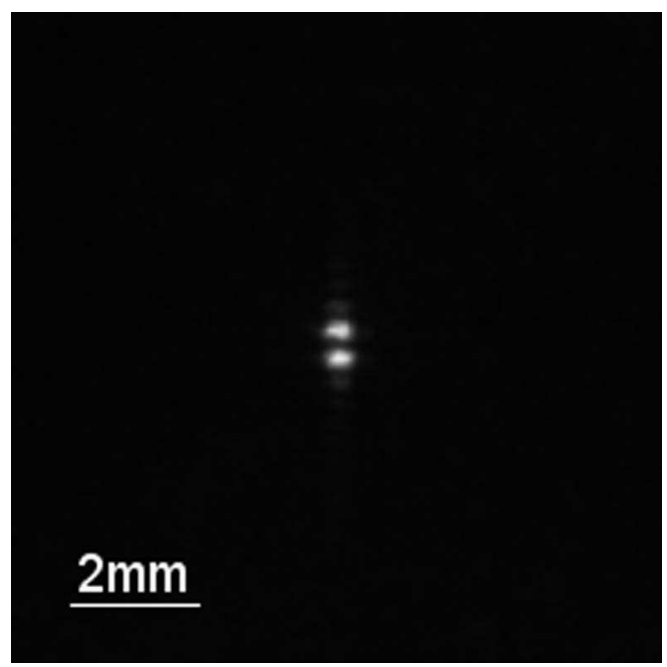

Fig. 9. Observation of the shape of order $(0,1)$. 


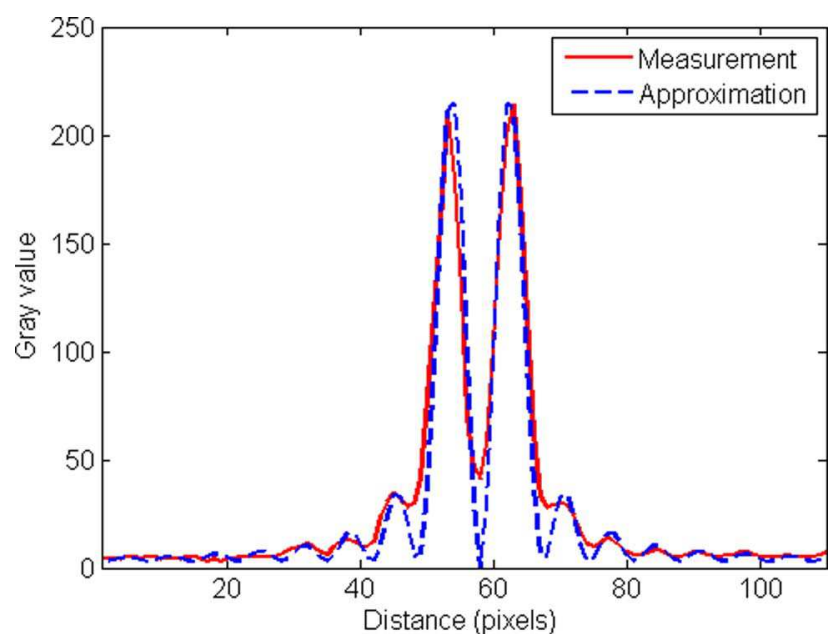

Fig. 10. (Color online) Vertical cross section of the image in Fig. 9 . The pixel pitch distance is $45 \mu \mathrm{m}$.

spacing in the plane $\Sigma_{2}$, we apply the same development as in Section 3. One may note that this situation is in fact trivial, because, as can be shown easily, sampling the continuous equivalent refractive lens of Eq. (1) at sampling points $j_{x} p$ after shifting it by $\lambda f n_{x} / \bar{p}$ changes only a constant in the sampled transmittance $t_{\Delta}$. Nevertheless, we assume here that the order centered at $\xi=0$ is $n=n_{X}$ and not $n=0$.

Then when $a=p$,
Defining the floor and ceil functions as follows,

$$
\begin{aligned}
& \forall x \in \mathbb{R}, \text { floor }(x)=\max \{n \in \mathbb{Z}, n \leq x\}, \\
& \forall x \in \mathbb{R}, \operatorname{ceil}(x)=\min \{n \in \mathbb{Z}, n \geq x\},
\end{aligned}
$$

it is seen from Eq. (23) that ceil $(2 p / a)$ diffraction patterns associated with diffraction orders are located within the main lobe of $\operatorname{sinc}(\xi a / \lambda f)$. We can approximate that these central orders have large efficiencies in comparison with other orders. However, one can once more note that these other orders are not weighted by $a A / 2 \pi \lambda f$, which means that the noncentral orders are more significant in relation to the central order(s) than if $a=p$ and the lens phase function were centered on an integer multiple of the diffracted order spacing in the focal plane $\Sigma_{2}$. Every order is here a sinc function. When $a=p$, two diffraction orders are significant: the orders floor $(-p X / \lambda f)$ and $\operatorname{ceil}(-p X / \lambda f)$.

\section{B. Examples}

As in Subsection 3.B, we want to illustrate the validity of the expressions describing the shapes and efficiencies of the diffraction orders determined via our approximations in Subsection 4.A. Therefore, we compare the results obtained via Eq. (18) with the analytical expressions describing the shape of the diffraction orders determined in Subsection 4.A. In

$$
\begin{gathered}
S_{n_{X}}(\xi) \simeq S_{A, n_{X}}(\xi)=p A \operatorname{sinc}(p \xi / \lambda f) \operatorname{sinc}(A \xi / \lambda f), \\
\forall n \neq n_{X}, S_{n}(\xi) \simeq S_{B, n}(\xi)=\left(p^{2} \operatorname{sinc}^{\prime}(p \xi / \lambda f) / 2 \pi i \lambda f\right) A^{2} \operatorname{sinc}^{\prime}[(\xi / \lambda f-X / \lambda f-n / p) A]
\end{gathered}
$$

and when $a \neq p$,

$$
\begin{aligned}
\forall n & \in \mathbb{Z}, S_{n}(\xi) \simeq S_{A, n}(\xi) \\
& =a \operatorname{sinc}\left(\frac{\xi}{\lambda f} a\right) A \operatorname{sinc}\left[\left(\frac{\xi}{\lambda f}-\frac{X}{\lambda f}-\frac{n}{p}\right) A\right]
\end{aligned}
$$

\section{Lens Decentering Not an Integer Multiple of the Diffracted Order Spacing}

Let us now consider the case in which the lens decentering in the pupil is not an integer multiple of the diffracted order spacing $1 / p$ in the focal plane. For every $n$, and if $\xi$ is close to $n \lambda f / p+X$, sinc $a \xi / \lambda f \neq 0$. Moreover, as $\left(H_{2}\right)$ implies $a A / 2 \pi \lambda f \ll 1$, then $S \simeq S_{A}$ :

$$
\begin{aligned}
\forall n & \in \mathbb{Z}, S_{n}(\xi) \simeq S_{A, n}(\xi) \\
& =a \operatorname{sinc}\left(\frac{\xi}{\lambda f} a\right) A \operatorname{sinc}\left[\left(\frac{\xi}{\lambda f}-\frac{X}{\lambda f}-\frac{n}{p}\right) A\right]
\end{aligned}
$$

this section, the pupil is fixed and the lens is decentered. We keep the same lens characteristics: $A=$ $4 \mathrm{~mm}, p=10 \mu \mathrm{m}, \lambda=500 \mathrm{~nm}, f=1 \mathrm{~m}$; so $\left(H_{2}\right)$ is clearly verified.

In Table 2, we simulate the case where $a=p$ and $X=\lambda f /(3 p)$. Here the lens decentering $X$ is not an integer multiple of the diffracted order spacing $\lambda f / p$ in the focal plane. According to Subsection 4.A, there are $\operatorname{ceil}(2 p / a)=2$ main orders in the focal plane: floor $(-p X / \lambda f)=-1$ and ceil $(-p X / \lambda f)=0$. Indeed, we can observe these two orders in Table 2 around $\xi=X=\lambda f / 3 p=16.7 \mathrm{~mm} \quad$ (order 0) and $\xi=X=\lambda f / 3 p-\lambda f / p=-33.3 \mathrm{~mm}$ (order -1). One can also notice that order 1 is more significant in Table $\underline{2}$ than in Table $\underline{1}$, which is, as we have seen

Table 2. Normalized Intensity of Orders $-1,0$ and 1 in the Focal Plane of a Decentered Pixelated Lens ${ }^{a}$

\begin{tabular}{lccc}
\hline & \multicolumn{3}{c}{ Order } \\
\cline { 2 - 4 } Quantity & -1 & 0 & 1 \\
\hline$\xi(\mathrm{mm})$ & -33.33 & 16.67 & 66.67 \\
Normalized intensity & 0.251 & 1 & $6.22 \times 10^{-2}$ \\
\hline${ }^{a} a=p, X=\lambda f / 3 p$. & & &
\end{tabular}




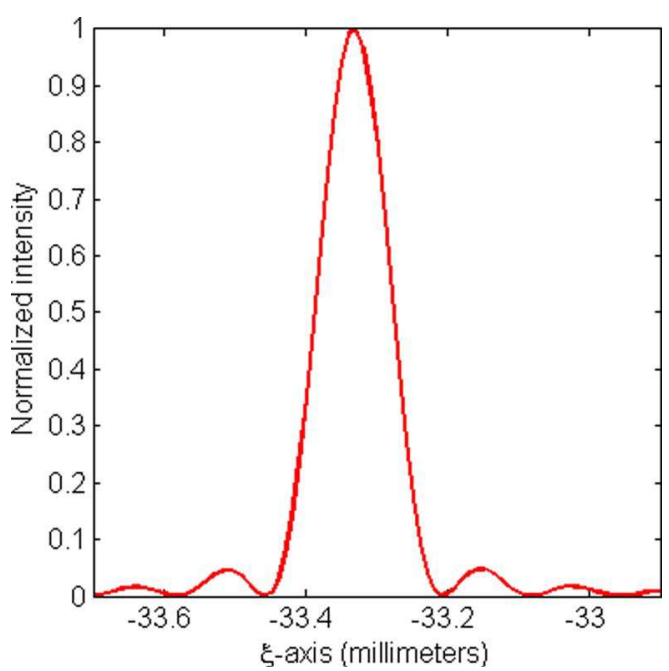

Fig. 11. (Color online) Order -1 in the focal plane of a decentered pixelated lens: $a=p, X=\lambda f /(3 p)$.

in Subsection 4.A, a consequence of the fact that this order is not weighted by $a A / 2 \pi \lambda f$ as in the case of the Table 1.

Now we zoom in around the order -1 in Fig. 11 . We compare the curves obtained via Eqs. (18) and (23). Here $\Delta=3.34 \times 10^{-3}$; then $S_{A,-1}$ is a good approximation of the shape of the order -1 .

Now we study the case where $a=p / 2$ and $X=2 \lambda f / 5 p$, represented in Fig. 12 . We superimposed the curve associated with $\operatorname{sinc}(a \xi / \lambda f)$ to verify that $\operatorname{ceil}(2 p / a)=4$ orders are located within the main lobe of $\operatorname{sinc}(a \xi / \lambda f)$ as expected. One can notice that in certain configurations, some orders located in the secondary lobes of $\operatorname{sinc}(\alpha \xi / \lambda f)$ can be brighter than some orders located in the main lobe.

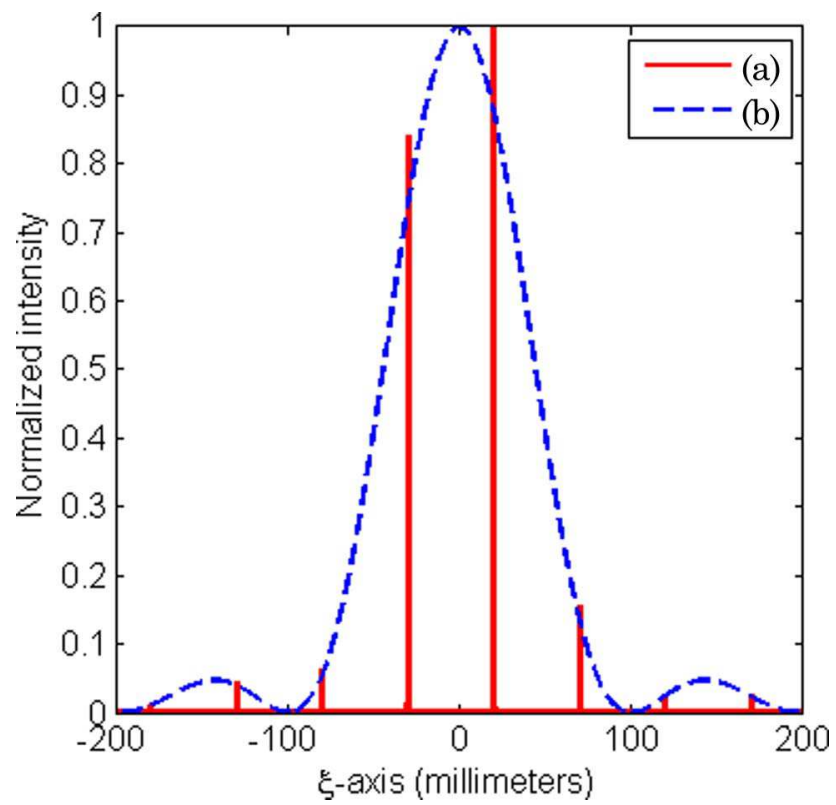

Fig. 12. (Color online) Normalized intensity in the focal plane of a decentered pixelated lens: $a=p / 2, X=2 \lambda f / 5 p$. (a) Curve associated with direct calculation; (b) curve associated with $\operatorname{sinc}(a \xi / \lambda f)$.
Let us sum up the results obtained in this section. When the center of the phase function is shifted with respect to optical axis along the $x$ axis, the brightest orders in the focal plane are located on both sides of the orthogonal projection of the lens center on $\xi$-axis. The number of significant orders depends on the ratio $a / p$. The shapes of all orders are generally similar to the PSF pattern of the equivalent refractive centered lens, except in the degenerate case where $a=p$ and the lens decentering is an integer multiple of the diffracted order spacing. Indeed, that case reduces to that of a centered lens.

\section{Conclusion}

We have analyzed the shape and efficiency of the diffraction orders observed in the focal plane of a pixelated lens when the pixel size is much smaller than the PSF of the ERL.

When the pixelated lens is centered, and when the pixel width a is equal to the pixel pitch $p$, we have shown that the central order whose shape is well known to be identical to that of the ERL is much brighter than the other orders and that the shapes of the other orders are approximately the derivative of this PSF. When the pixel width is sufficiently smaller than the pixel pitch, the shapes of all diffraction orders are similar to the PSF pattern of the ERL.

We have also shown that decentering the phase function of the lens along the $x$ or $y$ axis, with respect to the pupil, has a significant influence on the shape and efficiency of the diffraction orders.

We especially focused on pixelated lenses with square pixels and a square pupil, but our approach can be applied to different pupil and pixel shapes. Moreover, our study was about decentering the lens along the $x$ or $y$ axis and is trivially generalized to an arbitrary $2 \mathrm{D}$ decentering.

\section{Appendix A}

Starting again from Eq. (9),

$$
\begin{aligned}
S= & \sum_{\vec{j} \in(\mathbb{Z}, \mathbb{Z})} f_{\vec{j}}(\vec{\rho})=\sum_{\vec{j} \in(\mathbb{Z}, \mathbb{Z})} P(\overrightarrow{j p}) \exp \left(-2 i \pi \frac{\vec{j} p \cdot \vec{\rho}}{\lambda f}\right) \\
& \times \tilde{\Pi}_{2, a}\left(\frac{\vec{\rho}-\vec{j} p}{\lambda f}\right)
\end{aligned}
$$

$\left(H_{2}\right)$ implies, for any pixel $j$ inside the pupil, $\|\vec{j} p / \lambda f\| \leq 1 / p$.

Then

$$
\begin{aligned}
\tilde{\Pi}_{a}\left(\frac{\vec{\rho}-\vec{j} p}{\lambda f}\right) & \simeq \tilde{\Pi}_{a}\left(\frac{\xi}{\lambda f}, \frac{\eta}{\lambda f}\right)-\frac{j_{x} p}{\lambda f} \frac{\partial \tilde{\Pi}_{2, a}}{\partial \xi}\left(\frac{\xi}{\lambda f}, \frac{\eta}{\lambda f}\right) \\
& -\frac{j_{y} p}{\lambda f} \frac{\partial \tilde{\Pi}_{2, a}}{\partial \eta}\left(\frac{\xi}{\lambda f}, \frac{\eta}{\lambda f}\right) .
\end{aligned}
$$

Equation (A1) then becomes 


$$
\begin{gathered}
S(\xi)=\mathrm{FT}\left[P(x, y) \amalg_{p, p}(x, y)\right]_{(\xi / \lambda f, \eta / \lambda f)} \tilde{\Pi}_{2, a}\left(\frac{\xi}{\lambda f}, \frac{\eta}{\lambda f}\right) \\
-\frac{1}{\lambda f} \mathrm{FT}\left[x P(x, y) \amalg_{p, p}(x, y)\right]_{(\xi / \lambda f, \eta / \lambda f)} \frac{\partial \tilde{\Pi}_{2, a}}{\partial \xi}\left(\frac{\xi}{\lambda f}, \frac{\eta}{\lambda f}\right) \\
-\frac{1}{\lambda f} \operatorname{FT}\left[y P(x, y) \amalg_{p, p}(x, y)\right]_{(\xi / \lambda f, \eta / \lambda f)} \frac{\partial \tilde{\Pi}_{2, a}}{\partial \eta}\left(\frac{\xi}{\lambda f}, \frac{\eta}{\lambda f}\right) .
\end{gathered}
$$

FT is here the 2D Fourier transform.

Let us denote $\tilde{P}_{\xi}^{\prime}=\partial \tilde{P} / \partial \xi, \tilde{P}_{\eta}^{\prime}=\partial \tilde{P} / \partial \eta, \tilde{\Pi}_{2, a, \xi}^{\prime}=$ $\partial \tilde{\Pi}_{2, a} / \partial \xi$, and $\tilde{\Pi}_{2, a, \eta}^{\prime}=\partial \tilde{\Pi}_{2, a} / \partial \eta$ :

$$
\begin{aligned}
S(\xi)= & \left(\tilde{P} * \amalg_{(1 / p, 1 / p)}\right)_{\vec{\rho} / \lambda f} \tilde{\Pi}_{2, a}\left(\frac{\vec{\rho}}{\lambda f}\right) \\
& +\frac{1}{2 \pi i \lambda f}\left(\tilde{P}_{\xi}^{\prime} * \amalg_{(1 / p, 1 / p)}\right)_{\vec{\rho} / \lambda f} \tilde{\Pi}_{2, a, \xi}^{\prime}\left(\frac{\vec{\rho}}{\lambda f}\right) \\
& +\frac{1}{2 \pi i \lambda f}\left(\tilde{P}_{\eta}^{\prime} * \amalg_{(1 / p, 1 / p)}\right)_{\vec{\rho} / \lambda f} \tilde{\Pi}_{2, a, \eta}^{\prime}\left(\frac{\vec{\rho}}{\lambda f}\right) .
\end{aligned}
$$

This can also be written as

$$
\begin{aligned}
S(\xi)= & S_{A}(\xi)+S_{B, \xi}(\xi)+S_{B, \eta}(\xi) \\
= & \tilde{\Pi}_{2, a}\left(\frac{\vec{\rho}}{\lambda f}\right) \sum_{\vec{n} \in(\mathbb{Z}, \mathbb{Z})} \tilde{P}\left(\frac{\vec{\rho}}{\lambda f}-\frac{\vec{n}}{p}\right) \\
& +\frac{\tilde{\Pi}_{2, a, \xi}^{\prime}\left(\frac{\vec{\rho}}{\lambda f}\right)}{2 \pi i \lambda f} \sum_{\vec{n} \in(\mathbb{Z}, \mathbb{Z})} \tilde{P}_{\xi}^{\prime}\left(\frac{\vec{\rho}}{\lambda f}-\frac{\vec{n}}{p}\right) \\
& +\frac{\tilde{\Pi}_{2, a, \eta}^{\prime}\left(\frac{\vec{\rho}}{\lambda f}\right)}{2 \pi i \lambda f} \sum_{\vec{n} \in(\mathbb{Z}, \mathbb{Z})} \tilde{P}_{\eta}^{\prime}\left(\frac{\vec{\rho}}{\lambda f}-\frac{\vec{n}}{p}\right) .
\end{aligned}
$$

The $2 \mathrm{D}$ vectors $\vec{n}=\left(n_{\xi}, n_{\eta}\right)$, whose coordinates are integers, represent the diffraction orders.

$S_{A}$ is a replication of the lens pupil diffraction pattern in each diffraction order $n$ of the pixel grating, modulated by the pixel diffraction pattern.

$S_{B, \xi}\left(S_{B, \eta}\right)$ is a replication of the derivative of the lens pupil diffraction pattern along $\xi(\eta)$ in each diffraction order $\vec{n}$ of the pixel grating, modulated by the pixel diffraction pattern derivative in the $\xi$ direction ( $\eta$ direction).

$S_{A}, S_{B, \xi}$, and $S_{B, \eta}$ can be analyzed the same way as $S_{A}$ and $S_{B}$ in Sections $\underline{3}$ and $\underline{4}$.

\section{Appendix B}

We consider Eq. (1) in one dimension, when the pupil is translated along $x$, and $X$ is the coordinate of the new pupil center:

$$
t_{c}(x)=P(x) \exp \left(-i \pi(x-X)^{2} / \lambda f\right) .
$$

Then we use the Fraunhofer approximation for the central pixel diffraction at distance $f$,

$$
u_{0}(\xi)=-i \frac{U_{0}}{\lambda f} t_{c}(0) \exp \left(2 \pi i \frac{f}{\lambda}+i \pi \frac{\xi^{2}}{\lambda f}\right) \tilde{\Pi}_{1, a}\left(\frac{\xi}{\lambda f}\right)
$$

and for the diffraction by the pixel $j_{x}$ at distance $f$, we get, replacing $x$ with $j_{x} p$ in Eq. (B1),

$$
\begin{aligned}
u_{j_{x}}(\xi)= & -i \frac{U_{0}}{\lambda f} t_{c}\left(j_{x} p\right) \tilde{\Pi}_{1, a}\left(\frac{\xi-j_{x} p}{\lambda f}\right) \\
& \times \exp \left(2 \pi i \frac{f}{\lambda}+i \pi \frac{\left(\xi-j_{x} p\right)^{2}}{\lambda f}\right) .
\end{aligned}
$$

If we designate

$$
\begin{aligned}
f_{j_{x}}(\xi) \triangleq & P\left(j_{x} p\right) \exp \left(-2 i \pi \frac{j_{x} p \xi}{\lambda f}\right) \tilde{\Pi}_{1, a}\left(\frac{\xi-j_{x} p}{\lambda f}\right) \\
& \times \exp \left(\frac{2 i \pi X j_{x} p}{\lambda f}\right)
\end{aligned}
$$

then

$$
u_{j_{x}}(\xi)=-i \frac{U_{0}}{\lambda f} \exp \left[\frac{2 \pi i}{\lambda}\left(f+\frac{\xi^{2}}{2 f}\right)\right] \exp \left(-i \frac{\pi X^{2}}{\lambda f}\right) f_{j_{x}}(\xi)
$$

As the term $-\left(i U_{0} / \lambda f\right) \exp \left[(2 \pi i / \lambda)\left(f+\rho^{2} / 2 f\right)\right] \exp$ $\left(-i \pi X^{2} / \lambda f\right) f_{j_{x}}(\xi)$ is not pixel dependant, we focus on $\sum_{\vec{j} \in \mathbb{Z}} f_{\vec{j}}(\vec{\rho})$ :

$$
\begin{aligned}
S(\xi)= & \sum_{j_{x} \in \mathbb{Z}} f_{j_{x}}(\xi)=\sum_{j_{x} \in \mathbb{Z}} P\left(j_{x} p\right) \exp \left(-2 \pi i \frac{\xi j_{x} p}{\lambda f}\right) \\
& \times \tilde{\Pi}_{1, a}\left(\frac{\xi-j_{x} p}{\lambda f}\right) \exp \left(2 \pi i \frac{X j_{x} p}{\lambda f}\right) .
\end{aligned}
$$

Then to a first-order approximation

$$
\begin{aligned}
S(\xi)= & \mathrm{FT}\left[P(x) \amalg_{p}(x)\right]_{(\xi-X) / \lambda f} \tilde{\Pi}_{1, a}\left(\frac{\xi}{\lambda f}\right) \\
& -\frac{1}{\lambda f} \mathrm{FT}\left[x P(x) \amalg_{p}(x)\right]_{(\xi-X) / \lambda f} \tilde{\Pi}_{1, a}^{\prime}\left(\frac{\xi}{\lambda f}\right) .
\end{aligned}
$$

We notice that this expression would be obtained, in the centered case, with $t_{c}(x)=P(x) \exp \left(-i \pi x^{2} / \lambda f\right)$ $\exp (2 i \pi x X / \lambda f)$. The term $\exp (2 i \pi x X / \lambda f)$ represents a phase tilting, so we understand that within the context of our approximations, decentering this lens is equivalent to placing a thin prism before or after it.

Another way to write Eq. (B7) is

$$
\begin{aligned}
S(\xi)= & \left(\tilde{P} * \amalg_{1 / p}\right)_{(\xi-X) / \lambda f} \tilde{\Pi}_{1, a}\left(\frac{\xi}{\lambda f}\right) \\
& +\frac{1}{2 \pi i \lambda f}\left(\tilde{P} \prime * \amalg_{1 / p}\right)_{(\xi-X) / \lambda f} \tilde{\Pi}_{1, a}^{\prime}\left(\frac{\xi}{\lambda f}\right) .
\end{aligned}
$$


This can also be written as

$$
\begin{aligned}
S(\xi)= & S_{A}(\xi)+S_{B}(\xi) \\
= & \tilde{\Pi}_{1, a}\left(\frac{\xi}{\lambda f}\right) \sum_{n \in \mathbb{Z}} \tilde{P}\left(\frac{\xi}{\lambda f}-\frac{X}{\lambda f}-\frac{n}{p}\right) \\
& +\frac{\tilde{\Pi}_{1, a}^{\prime}(\xi / \lambda f)}{2 \pi i \lambda f} \sum_{n \in \mathbb{Z}} \tilde{P}_{\prime}\left(\frac{\xi}{\lambda f}-\frac{X}{\lambda f}-\frac{n}{p}\right) .
\end{aligned}
$$

Then, if $P(x)=\Pi_{1, A}(x)$, one obtains Eqs. (20).

\section{References and Notes}

1. D. M. Cottrell, J. A. Davis, T. R. Hedman, and R. A. Lilly, "Multiple imaging phase-encoded optical elements written as programmable spatial light modulators," Appl. Opt. 29, 2505-2509 (1990).

2. E. Carcolé, J. Campos, I. Juvells, and S. Bosch, "Diffraction efficiency of low-resolution Fresnel encoded lenses," Appl. Opt. 33, 6741-6746 (1994).
3. E. Carcolé, J. Campos, and S. Bosch, "Diffraction theory of Fresnel lenses encoded in low-resolution devices," Appl. Opt. 33, 162-174 (1994).

4. E. Carcolé, J. Campos, I. Juvells, and J. R. de F. Moneo, "Diffraction theory of optimized low-resolution Fresnel encoded lenses," Appl. Opt. 34, 5952-5960 (1995).

5. V. Arrizon, E. Carreon, and L. A. Gonzalez, "Self-apodization of low-resolution pixelated lenses," Appl. Opt. 38, 5073-5077 (1999).

6. G. J. Swanson, "The theory and design of multi-level diffractive optical elements," Binary Optics Technology, MIT Tech. Rep. 854 (MIT, 1989)

7. G. J. Swanson"Theoretical limits on the diffraction efficiency of multilevel diffractive optical elements," in Binary Optics Technology, MIT Tech. Rep. 914 (MIT, 1991).

8. This is compatible with a centered pupil of size $N \times N$ pixels only if $N$ is an odd integer. But the extension to even values of $N$ would be straightforward. However, the parity of $N$ is an inessential factor in the present study and we shall not develop it in any detail.

9. S. Wang, E. Barnabeu, and J. Alda, "Unified and generalized Fresnel numbers," Opt. Quantum Electron. 24, 1351-1358 (1992). 